\title{
Notoginsenoside R2 induces colonic microvascular injuries via regulating the Rap1GAP/PI3K/Akt signaling pathway
}

\author{
Ping Tao ${ }^{1 \wedge}$, Jiang Lin $^{2} \wedge$, Beiping Zhang ${ }^{1 \wedge}$, Shiying Wang ${ }^{1,3} \wedge$ \\ ${ }^{1}$ Department of Gastroenterology, The Second Affiliated Hospital of Guangzhou University of Chinese Medicine, Guangzhou, China; ${ }^{2}$ Department \\ of Gastroenterology, Longhua Hospital, Shanghai University of Traditional Chinese Medicine, Shanghai, China; ${ }^{3}$ Science and Technology \\ Innovation Center, Guangzhou University of Chinese Medicine, Guangzhou, China. \\ Contributions: (I) Conception and design: S Wang, J Lin; (II) Administrative support: B Zhang, J Lin; (III) Provision of study materials or patients: S \\ Wang, P Tao; (IV) Collection and assembly of data: P Tao; (V) Data analysis and interpretation: S Wang, P Tao; (VI) Manuscript writing: All authors; \\ (VII) Final approval of manuscript: All authors. \\ Correspondence to: Shiying Wang, PhD. Science and Technology Innovation Center, Guangzhou University of Chinese Medicine, 12 Jichang Road, \\ Baiyun District, Guangzhou 510405, China. Email: yuchao387653@126.com.
}

Background: Notoginsenoside R2 (NGR2) is an important active saponin molecule of Panax Notoginseng $(\mathrm{PN})$, but its effect on regulating angiogenesis is unclear. Here, we investigated the role of NGR2 in angiogenesis in vivo and in vitro.

Methods: NGR2 was administered to rats by intragastric administration for 7 days. The colonic histopathology and microvessel density (MVD) were observed and evaluated under an inverted microscope. The colonic mucosal permeability (MP) and vascular permeability (VP) were evaluated by measuring the transmittance of FD-4 and the vascular leakage of Evans blue, respectively. The serum IL-2, TNF- a, IL-4, IL-10, VEGFA165, and VEGFA121 levels were detected with ELISA. In vitro, pHUVECs were cultured and treated with NGR2. The mechanism of NGR2 in angiogenesis was assessed by VEGFA165, LY294002, silencing, and overexpression plasmids of Rap1GAP in vitro. The cell viability, cell proliferation, tube formation, and concentration of intracellular pyruvate and lactic acid of pHUVECs were measured after $24 \mathrm{~h}$. The expression of proteins was detected with western blotting.

Results: NGR2 could significantly induce inflammatory injuries in the colonic mucosa and microvessels. Both MP and VP in rats treated with NGR2 increased in proportion to the serum VEGFA165 and VEGFA121 level, the ratio of VEGFA165/VEGFA121, and the concentration of intracellular pyruvate and lactic acid. In vitro, NGR2 reduced cell viability, proliferation, and tube formation, and enhanced the intracellular glycolysis of pHUVECs. Furthermore, the cell viability, proliferation, and tube formation of pHUVECs were inhibited by NGR2 via blocking the Rap1GAP/PI3K/Akt signaling pathway.

Conclusions: NGR2 could induce colonic mucosal microvascular injuries and promoted the intracellular glycolysis of pHUVECs via blocking the Rap1GAP/PI3K/Akt signaling pathway.

Keywords: Notoginsenoside R2 (NGR2); glycolysis; angiogenesis; PI3K/Akt signaling pathway

Submitted Oct 25, 2021. Accepted for publication Nov 29, 2021.

doi: $10.21037 / \mathrm{atm}-21-5898$

View this article at: https://dx.doi.org/10.21037/atm-21-5898

\footnotetext{
^ ORCID: Ping Tao, 0000-0002-5914-8478; Jiang Lin, 0000-0001-8524-6103; Beiping Zhang, 0000-0003-2979-9226; Shiying Wang, 00000002-1005-0381.
} 


\section{Introduction}

Panax Notoginseng $(\mathrm{PN})$ has been used traditionally for the treatment of traumatic injuries in East Asia for more than 2,000 years. The formation of granulation tissue during the repair of traumatic injuries is the result of the controllable regulation of angiogenesis, including promoting angiogenesis and anti-angiogenesis (1), which have been shown to be regulated by PN (2). Our previous research found that PN could significantly promote angiogenesis and reduce vascular inflammatory injuries $(3,4)$. Indeed, some saponins in $\mathrm{PN}$, which are important active chemical components including ginsenosides and notoginsenosides, have appeared to lack toxicity in both animals and humans (5). For example, notoginsenoside, such as NGR1, NGFc, and NGRg1, had the effect of promoting angiogenesis, protecting the blood vessels from injuries, and inhibiting inflammation $(6,7)$. However, the antiangiogenesis effect and active ingredients of $\mathrm{PN}$ have been seriously neglected in previous studies.

Notoginsenoside R2 (NGR2) is an active saponin molecule extracted from the root of $P N$. Previous studies showed that it reduced A25-35-induced neuronal apoptosis and inflammation (8), and stimulated the growth of bone marrow nucleated cells and hematopoietic stem cells, as well as burst-forming unit-erythroid and colony-forming unit granulocyte-monocyte colony expansion (9). However, the role of NGR2 in angiogenesis remains unexplored, which is precisely the purpose of this study.

The Ras-related protein 1-GTPase-activating protein 1 (Rap1GAP)/PI3K/Akt signaling pathway is an important signal pathway regulating the cell proliferation of vascular endothelial cells (VECs), which can be activated by vascular endothelial growth factor A (VEGFA). Activating the VEGFA/Rap1GAP/PI3K/Akt signaling pathway could significantly promote the cell proliferation of human umbilical vein endothelial cells (HUVECs) and reduce inflammation $(10,11)$. Our previous studies also found that the VEGF/Rap1GAP signaling pathway had a critical role in regulating the cell proliferation of VECs, as blocking the VEGF/Rap1GAP signaling pathway could significantly increase colonic mucosal inflammatory injuries in rats via up-regulating the expression of thrombospondin 1 (TSP1) (12). TSP1 is a critical protein involved in increasing vascular injuries and promoting vascular remodeling $(13,14)$. Our results in previous studies showed that $\mathrm{PN}$ could significantly regulate the secretion of VEGFA165 and VEGFA121 and down-regulate the expression of TSP1 protein in colonic mucosa (3). However, the regulatory effect of NGR2 on the Rap1GAP/PI3K/ Akt signaling pathway is unclear. Here, we investigated the role of NGR2 in angiogenesis in vivo and in vitro to further understand the anti-angiogenesis effect of PN. The findings suggest that PN may have potential vascular toxicity except for promoting angiogenesis.

We present the following article in accordance with the ARRIVE reporting checklist (available at https://dx.doi. org/10.21037/atm-21-5898).

\section{Methods}

\section{Cell culture and animals}

Primary human umbilical vein endothelial cells (pHUVECs) were obtained from ATCC (Maryland, USA) and maintained in vascular cell basal medium (ATCC) at $37{ }^{\circ} \mathrm{C}$ in a humidified atmosphere of $95 \%$ air and $5 \% \mathrm{CO}_{2}$.

Sprague Dawley (SD) rats (120-140 g) were purchased from Shanghai SLAC Laboratory Animal Co. Ltd. (Shanghai, China). The care and treatment of mice were in accordance with institutional guidelines. All animals used in the present study were housed in a pathogen-free environment, and animal experiments were approved by the Institutional Animal Care and Use Committee of Shanghai University of TCM (No. SZY201612006), in compliance with the Institutional Animal Care and Use Committee of Shanghai University of TCM's guidelines for the care and use of animals. After 1 week of adaptive feeding, the study begun when the rats behaved normally.

\section{Reagents}

Formamide (purity was above 99\%) and Evans Blue (no: 295876 , dye content was above $75 \%$ ) were purchased from Sigma Chemical. Co. (Sigma-Aldrich, MO, USA). NGR2 was obtained from Shanghai R\&D Center for Standardization of Chinese Medicines (Shanghai, China). Enzyme-linked immunosorbent assay (ELISA) kits (IL-10, IL-4, IL-6, and TNF-a) were from R\&D Systems (R\&D, USA). VEGFA121 (no: SEB851Ra) and VEGFA (no: SEA143Ra) ELISA kits were purchased from Cloud-Clone Co. Anti-VEGFR2 antibody (ab11939), anti-VEGFR2 
(phospho Y1214) antibody (ab5475), and anti-Rap1GAP antibody (ab32373) were purchased from Abcam Co. (London, United Kingdom). Anti-VEGFR2 (phospho Y1175) antibody (AF4426) was from Affinity Biosciences (USA). Human VEGFA165 protein (\#8065), LY294002 (PI3K inhibitor, \#9901), anti-PI3K antibody (\#4249), phospho-PI3K (\#4228), Akt (\#4691), phospho-Akt (\#13038 and \#4060) antibodies, goat anti-mouse antibody, and goat anti-rabbit antibody were from Cell Signaling Technology (USA). Anti-CD31 antibody (sc-376764) and anti-TSP1 antibody (sc-393504) were from Santa Cruz Biotechnology Co. (Santa Cruz, CA, USA). Human Rap1GAP gene silencing plasmid (designed by targeting the NM_001145658 gene) and human Rap1GAP gene overexpression plasmid (targeting the NM_001145658 gene) were constructed and synthesized by GK Gene (Shanghai, China). Lipofectamine 2000 Transfection Reagent (\#11668019) was purchased from Life Technologies Co. (Waltham, MA).

\section{NGR2 treatment in vivo}

NGR2 (5.0 10.0, and 20.0 $\mu \mathrm{M}$ ) was administered to normal rats once a day by intragastric administration (i.g., $\mathrm{n}=8$ ). In the normal control group $(\mathrm{n}=8)$, normal saline was administered to the rats i.g. once a day. After 7 days, the rats were anesthetized with $180 \mu \mathrm{L} 3 \%$ sodium pentobarbital per $100 \mathrm{~g}$. Blood was collected from the abdominal aorta and the colon tissues were obtained. The colon tissues were formaldehyde-fixed, paraffin-embedded, and stained with hematoxylin and eosin (HE). A protocol was prepared before the study without registration.

\section{pHUVECs treated with LY294002, VEGFA165, and NGR2}

pHUVECs were treated with VEGFA165 $(1.0 \mathrm{nM})$, LY294002 $(50 \mu \mathrm{M})$, and NGR2 (5.0, 10.0, and $20.0 \mathrm{nM})$ for $24 \mathrm{~h}$ or in combination. The medium was removed and the adherent cells were washed 3 times with phosphatebuffered saline (PBS) to eliminate any residual LY294002 and NGR2. Fresh DMEM without fetal bovine serum (FBS) was added to the cells for $3 \mathrm{~h}$. This conditioned medium $(\mathrm{CM})$ was centrifuged for $15 \mathrm{~min}$ at 5,000 $\times$ g, passed through a $0.2-\mu \mathrm{m}$ filter, and incubated for $2 \mathrm{~h}$ at $37^{\circ} \mathrm{C}$ with the remaining medium removed.

\section{Histopathology, microvessel density (MVD), vascular permeability (VP), mucosal permeability (MP), cell viability assay, cell proliferation assay, tube formation assay, plasmid construction and cell transfection, and western blotting}

Histopathology, MVD, cell viability assay, cell proliferation assay, tube formation assay, plasmid construction and cell transfection, and western blotting were carried out as previously described (12). The VP and MP in the colon were detected as previously described $(3,15)$. All assays were performed at least 3 times.

\section{Metabolic substrate assay}

After treatment, the content of pyruvate and lactate in cell lysates was measured using the NADH optical test as previously described (16). For determining the intracellular pyruvate and intracellular lactate levels, $100 \mu \mathrm{L}$ of lysate from $1 \times 10^{6}$ trypsinized cells was prepared with a hypotonic buffer $(20 \mathrm{mM}$ HEPES, $0.5 \mathrm{mM} \mathrm{CaCl}$, $1 \mathrm{mM} \mathrm{MgCl}{ }_{2}$ ). Following centrifugation at 13,000 $\times \mathrm{g}$, $100 \mu \mathrm{L}$ of supernatant was collected and adjusted to $\mathrm{pH}$ $6.5-8.5$ by adding $64.6 \mu \mathrm{L}$ of ice-cold $2 \mathrm{M} \mathrm{KOH}$ for $20 \mathrm{~min}$. The resulting precipitate was centrifuged and the supernatant was collected. After incubating for $30 \mathrm{~min}$ at room temperature, the change in fluorescence was measured at the excitation and emission wavelengths of 355 and $460 \mathrm{~nm}$, respectively (Victor 3, Perkin Elmer). Pyruvate and lactate concentrations were calculated from a reference curve. Subsequently, the intracellular lactate concentration was calculated based on the concentration of protein in the cell lysate $(1 \mu \mathrm{g} / \mu \mathrm{L})$.

\section{Statistical analysis}

Data were presented as the mean \pm SEM. One-way ANOVA or general linear model with repeated measures was used to analyze data sets with 3 or more groups, and the least significant difference post hoc test was used for multiple comparisons. Student's $t$-test was used to analyze data sets with two groups. $\mathrm{P}<0.05$ was considered significant. 


\section{Results}

NGR2 induced microvascular and mucosal injuries in the rat colons

SD rats were divided into the control group (NS, iv), low-dose group (5.0 $\mu \mathrm{M}$ NGR2, iv), medium-dose group (10.0 $\mu$ M NGR2, iv), and high-dose group $(20.0 \mu \mathrm{M}$ NGR2, iv). After 7 days of treatment, obvious colonic mucosal inflammation and vascular injuries were found in the colonic mucosa of the high-, medium-, and lowdose groups compared with the control group (Figure 1A). Meanwhile, VP was increased greatly in both the highdose group and medium-dose group, along with increased MP (Figure $1 B, 1 C$ ). In addition, the serum contents of pro-inflammatory cytokines (IL- 6 and TNF- $\alpha$ ) were significantly higher, and serum IL-4 and IL-10 were significantly lower in the high-dose group and mediumdose group than those in the control group (Figure 1D-1G). The results showed that NGR2 could lead to vascular and mucosal inflammatory injuries, with increased VP in rat colons.

\section{NGR2 increased hypoxia in the colon mucosa with an increased ratio of serum VEGFA165/VEGFA121 and increased protein expression of Rap1GAP and TSP1}

With the colonic mucosal vascular injuries increased, the protein expression levels of Rap1GAP and TSP1 in the colon mucosa were significantly higher in the high-dose group and medium-dose group than in the control group. However, there were no significant differences in the protein expression of VEGFR2, VEGFR2 (p1175), and VEGFR2 (p1214) between the control group and treatment groups (Figure 2A). Meanwhile, the serum contents of VEGFA165 and VEGFA121 and the ratio of VEGFA165/ VEGFA121 were significantly higher in the high-dose group and medium-dose group than in the control group (Figure 2B,2C).

In addition, the protein expression of HIF- $1 \alpha$ and the concentration of intracellular pyruvate and lactic acid were significantly increased in the colon mucosa in the high-dose group and medium-dose group (Figure $2 A, 2 D, 2 E$ ).

The results suggested that the microvascular injuries of the colon mucosa in rats treated with NGR2 aggravated the hypoxia in the colon mucosa, and might be related to the increased serum ratio of VEGFA165/VEGFA121 and the protein expression of Rap1GAP and TSP1.

\section{NGR2 promoted intracellular glycolysis and inbibited the} cell proliferation and cell viability of pHUVECs

To clarify the mechanism of NGR2 in inducing vascular injury in colon tissues, the effect of NGR2 on regulating the cell viability of pHUVECs was measured at 24 and $48 \mathrm{~h}$. NGR2 (from 0 to $50 \mathrm{nM}$ ) inhibited the cell viability in a concentration-dependent manner (Figure $3 A$ ). The IC50 of NGR2 was $25.94 \mathrm{nM}$ at $24 \mathrm{~h}$. Therefore, in the following experiments, the concentrations used to treat pHUVECs were 20.0, 10.0, and 5.0 nM. Compared with the control group, the cell viability and the cell proliferation of the groups treated with NGR2 $(10.0$ and $20.0 \mu M)$ were significantly inhibited at $24 \mathrm{~h}$ (Figure 3B,3C).

In addition, the concentrations of intracellular pyruvate and lactic acid were significantly increased in pHUVECs in the high-dose group and medium-dose group (Figure 3D,3E).

The results showed that NGR2 could inhibit the cell viability and proliferation of pHUVECs and promote intracellular glycolysis.

\section{NGR2 inbibited the cell proliferation and tube formation of pHUVECs induced by VEGFA165}

VEGFA165, one of the VEGFA isoforms that promotes the cell proliferation of pHUVECs, was used to explore the mechanism of NGR2 in inhibiting angiogenesis. As shown in Figure $4 A$ and Figure $4 B$, cell viability and proliferation were increased by VEGFA165 (1.0 nM). When pHUVECs were co-treated with VEGFA165 and NGR2 $(10.0 \mathrm{nM})$, cell viability and proliferation were significantly inhibited. In addition, the number of tube formations also obviously decreased in the VEGFA165 + NGR2 group compared to the VEGFA165 group. However, there was no significant difference in the tube formation between the NGR2 group and the VEGFA165 + NGR2 group (Figure 4C,4D).

The results demonstrated that NGR2 may counteract the role of VEGF in the regulation of cell viability and proliferation.

\section{NGR2 inbibited the cell proliferation of pHUVECs via targeting Rap1GAP}

To explore the role of Rap1GAP in regulating angiogenesis, a Rap1GAP interference plasmid was used. The expression of the Rap1GAP gene in pHUVECs was up-regulated by 
A

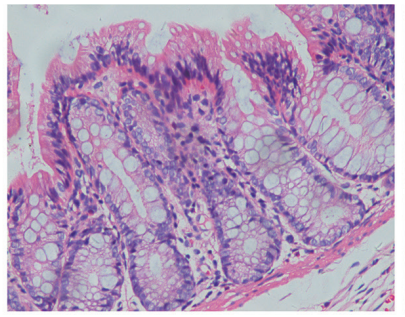

Control

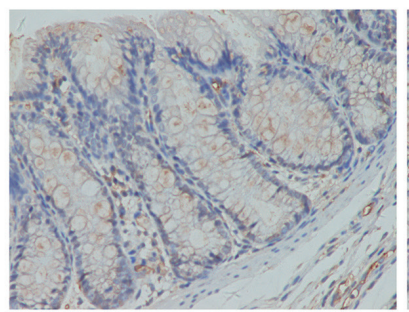

Control

B

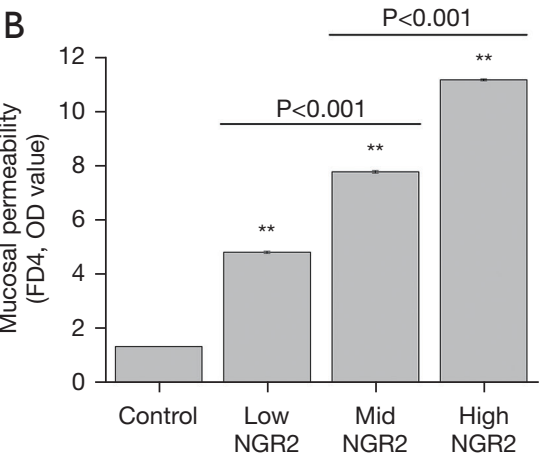

E

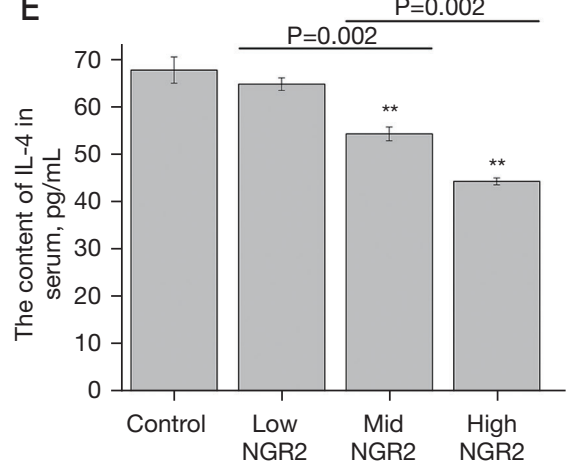

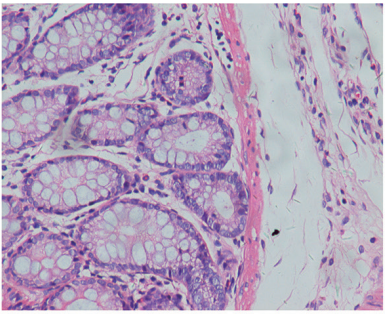

Low NGR2

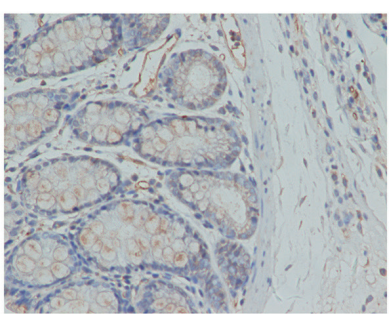

Low NGR2

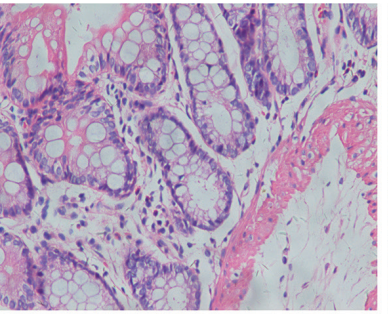

Mid NGR2

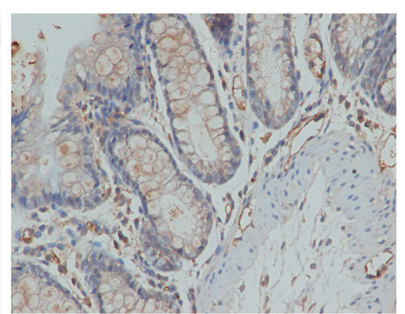

Mid NGR2

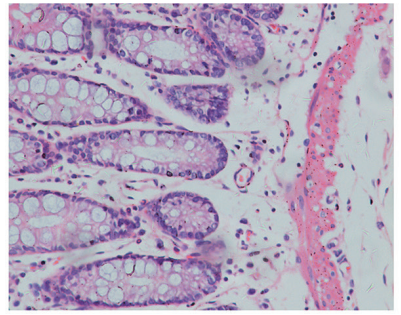

High NGR2

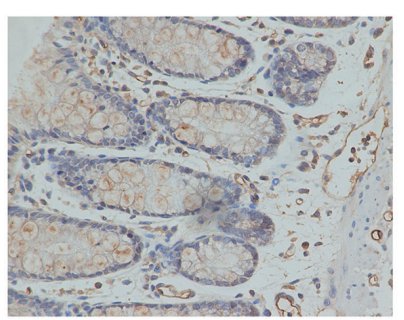

High NGR2
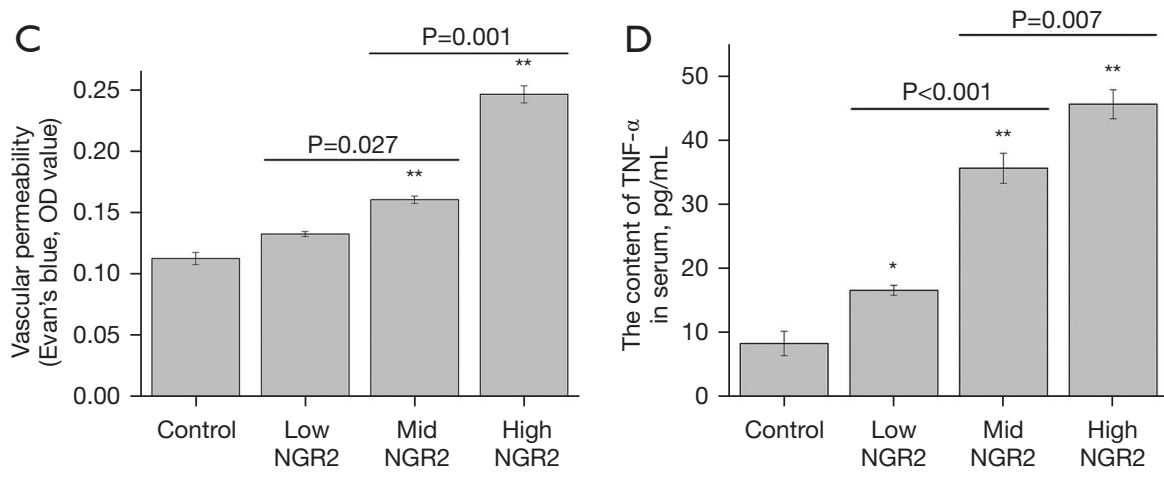

$\mathrm{F}$
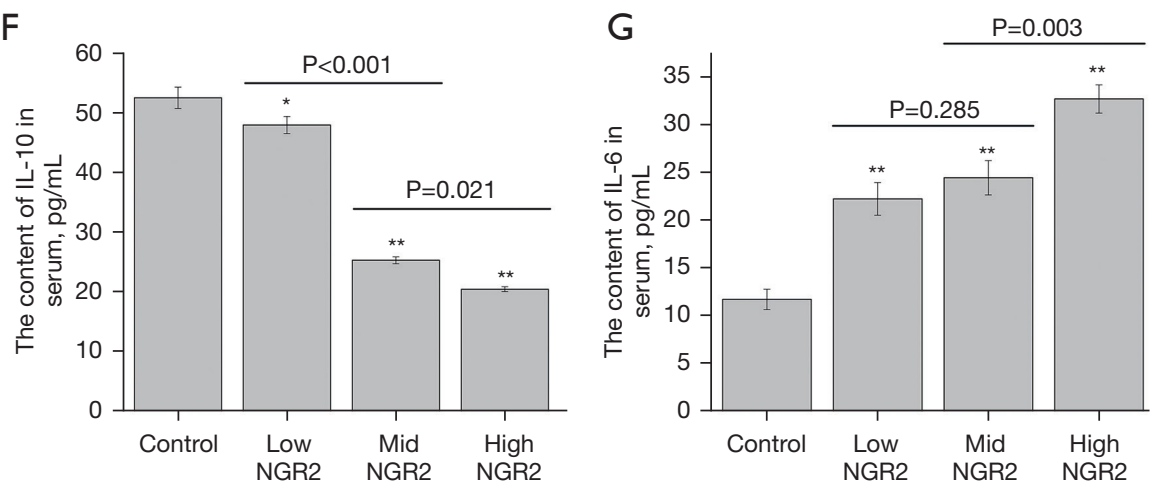

Figure 1 NGR2 induced microvascular and mucosal injuries in the colons of rats. (A) Colonic mucosal inflammation (HE) and vascular injury (400x); (B,C) Vascular permeability and mucosal permeability in the colonic mucosa; (D-G) the serum contents of IL-6, TNF- $\alpha$, IL-4, and IL-10. The data are means \pm SEM of 3 independent experiments performed in triplicate. One-way ANOVA and Student's $t$-test $\left({ }^{*} \mathrm{P}<0.05\right.$, ${ }^{* *} \mathrm{P}<0.001$ vs. control). 
A

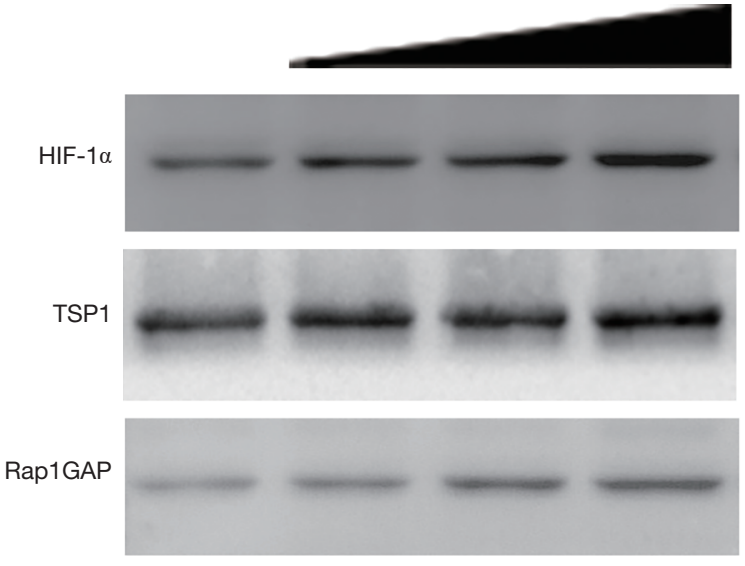

VEGFR2 (p1214)

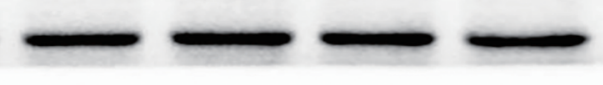

VEGFR2 (p1175)

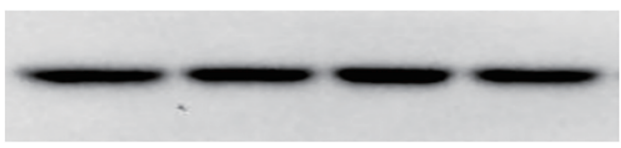

VEGFR2

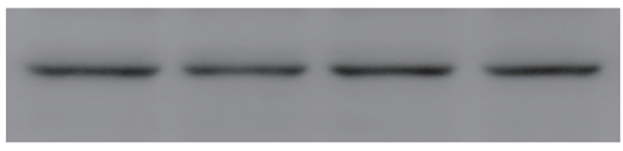

$\beta$-actin

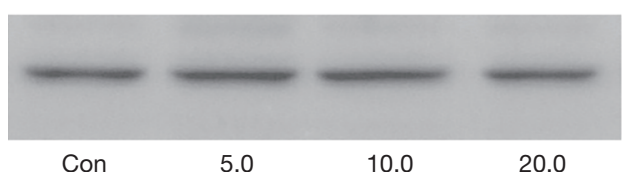

D

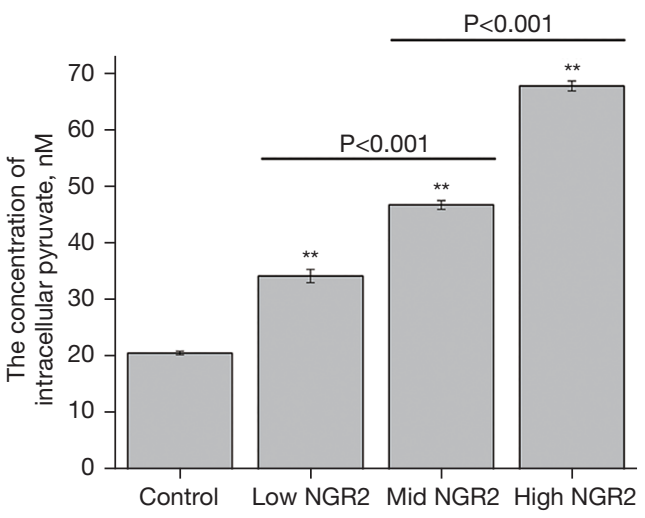

B

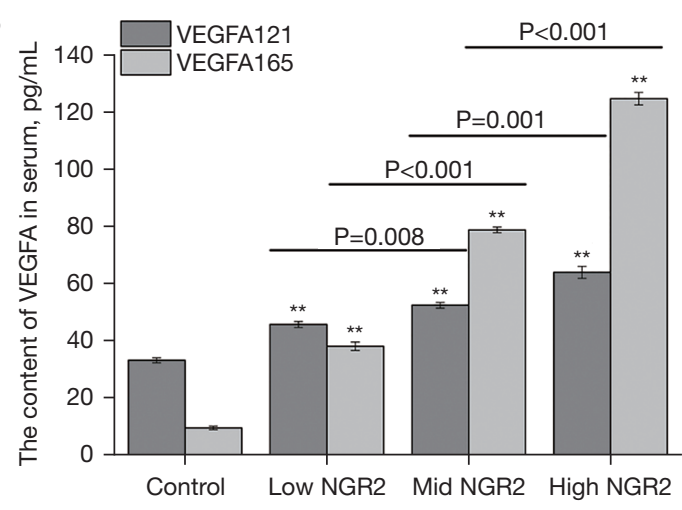

C

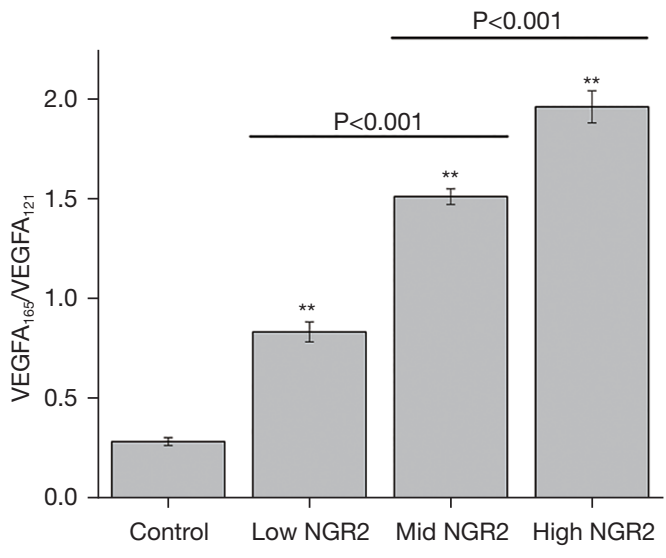

$E$

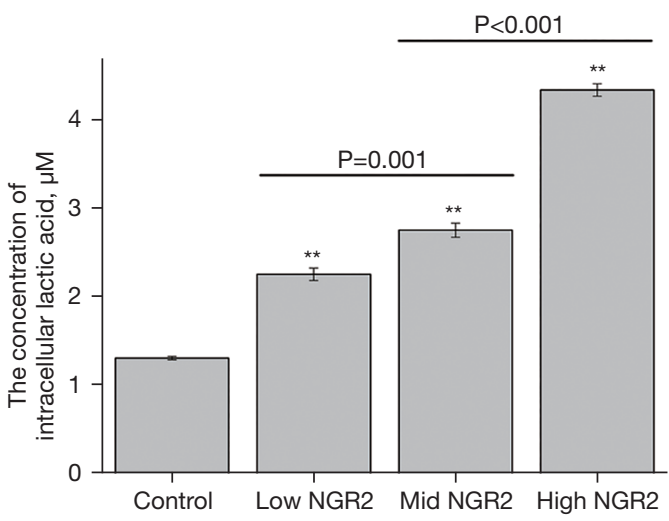

Figure 2 NGR2 increased hypoxia in the colon mucosa with an increased ratio of serum VEGFA165/VEGFA121 and increased protein expression of Rap1GAP and TSP1. (A) The protein expression levels of VEGFR2, VEGFR2 (p1175), VEGFR2 (p1214), Rap1GAP, TSP1, and HIF-1 $\alpha$ in the colon mucosa; (B,C) the serum contents of VEGFA165 and VEGFA121 and the ratio of VEGFA165/VEGFA121; (D,E) the concentration of intracellular pyruvate and lactic acid in the colonic mucosa. The data are means \pm SEM of 3 independent experiments performed in triplicate. One-way ANOVA and Student's $t$-test ${ }^{* *}, \mathrm{P}<0.001 v s$. control). 

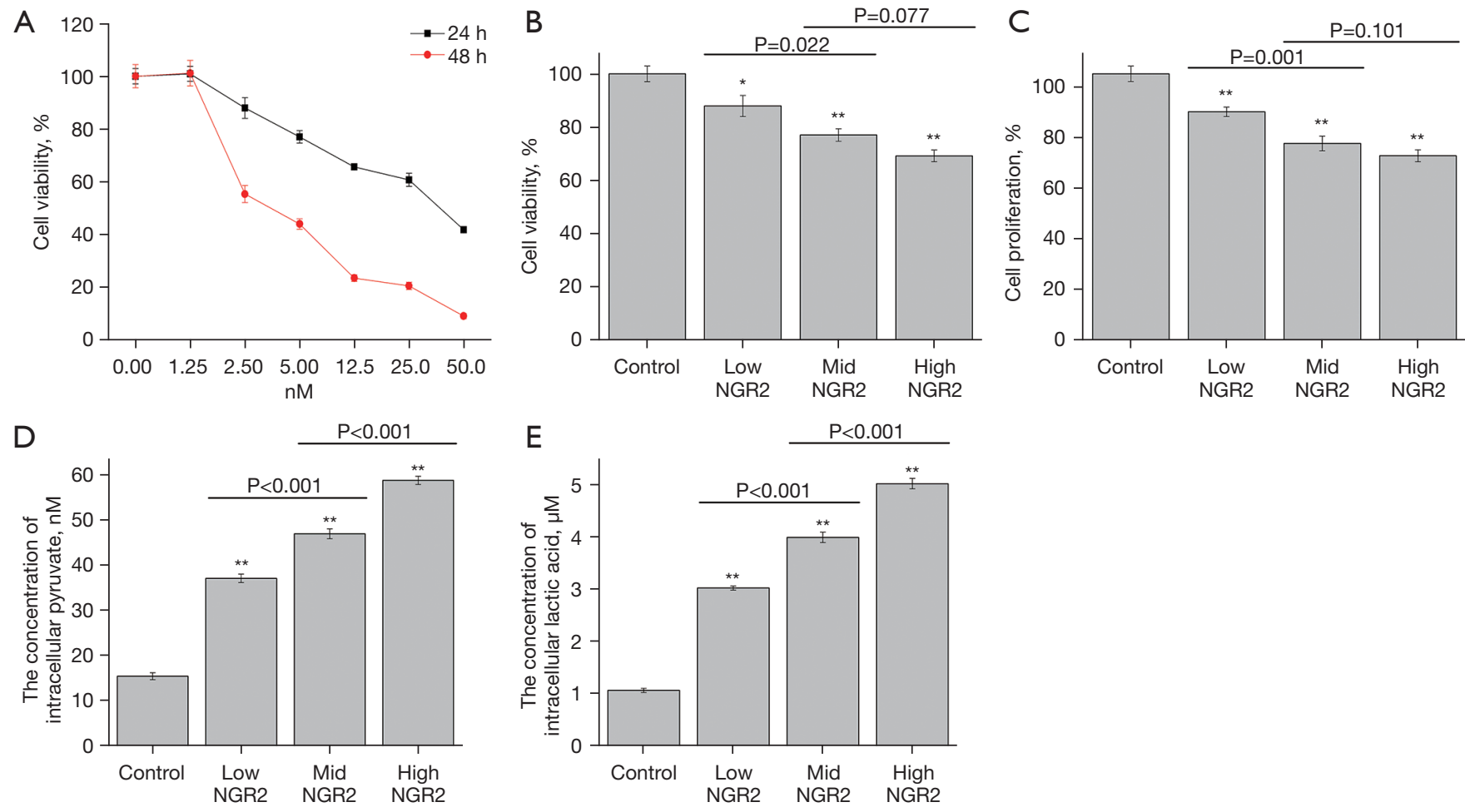

Figure 3 NGR2 promoted intracellular glycolysis and inhibited the cell proliferation and cell viability of pHUVECs. (A) The effect of NGR2 on regulating the cell viability of pHUVECs was measured at 24 and $48 \mathrm{~h}$ (IC50 of NGR2 was $25.94 \mathrm{nM}$ at 24 h); (B,C) the cell viability and cell proliferation of pHUVECs treated with NGR2; (D,E) the concentration of intracellular pyruvate and lactic acid in pHUVECs. The data are means \pm SEM of 3 independent experiments performed in triplicate. One-way ANOVA and Student's $t$-test $\left({ }^{*} \mathrm{P}<0.05,{ }^{* *} \mathrm{P}<0.001\right.$ vs. control).

the Rap1GAP overexpression plasmid (Rap1GAP pler plasmid, Rap1GAP-ov) (Figure 5A). Furthermore, VEGFA165 could down-regulate the protein expression of Rap1GAP in pHUVECs (Figure 5B). Meanwhile, the cell viability and cell proliferation of pHUVECs increased in the VEGFA165 group compared with the control group (Figure 4A,4B).

The protein expression of Rap1GAP in pHUVECs in the groups treated with NGR2 (10.0 nM), VEGFA165 $(1.0 \mathrm{nM})+$ NGR2, VEGFA165 (1.0 nM) + Rap1GAP-ov, and Rap1GAP-ov + NGR2 + VEGFA165 was significantly up-regulated compared with the control and VEGFA165 group, and the expression of TSP1 was also up-regulated. Additionally, the expression of TSP1 in the group treated with VEGFA165 was also down-regulated compared with the control group (Figure $5 B$ ).

Meanwhile, cell viability and cell proliferation in the groups treated with NGR2 (10.0 nM), VEGFA165 $(1.0 \mathrm{nM})+$ NGR2, VEGFA165 (1.0 $\mathrm{nM})+$ Rap1GAP-ov, Rap1GAP-ov + NGR2 + VEGFA165, and Rap1GAP-ov were inhibited compared with the control and VEGFA165 treatment group, with increased concentrations of intracellular pyruvate and lactic acid. There were no significant differences in cell viability, cell proliferation, and the concentrations of intracellular pyruvate and lactic acid between the groups treated with VEGFA165 + Rap1GAP$o v+$ NGR2 and Rap1GAP-ov + VEGFA165 or between the groups treated with VEGFA165 + NGR2 and NGR2 (Figure 5C-5F).

The Rap1GAP silencing plasmid (Rap1GAP plasmid, Rap1GAP-si) was used to silence the expression of Rap1GAP in pHUVECs to explore the mechanism of NGR2 in regulating angiogenesis. We found that Rap1GAP-si 3 could silence Rap1GAP expression (Figure $5 G$ ). In the following experiments, Rap1GAP-si 3 was chosen as the silencing plasmid.

Furthermore, the protein expression levels of Rap1GAP and TSP1 in pHUVECs in the groups treated with Rap1GAP-si, NGR2 + Rap1GAP-si, and VEGFA165+ 

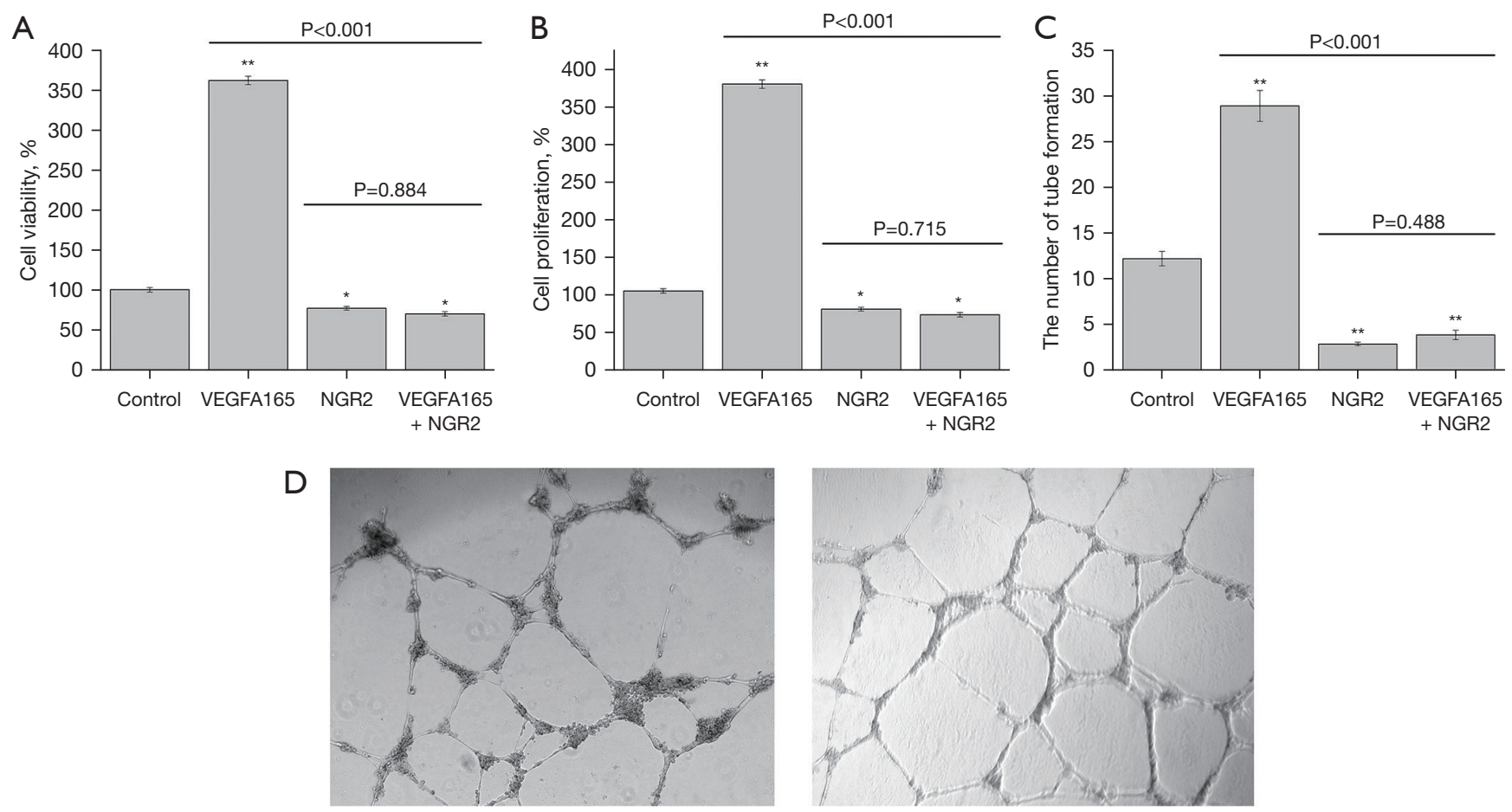

Control

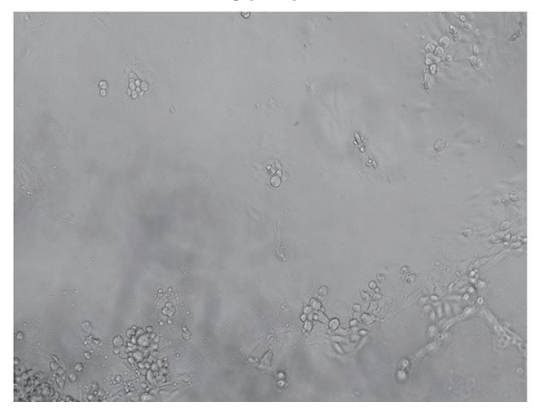

NGR2

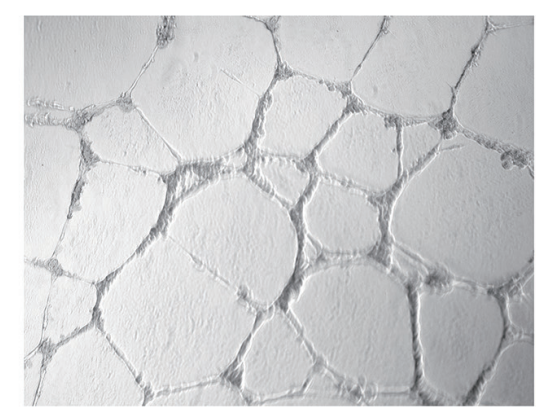

VEGFA165

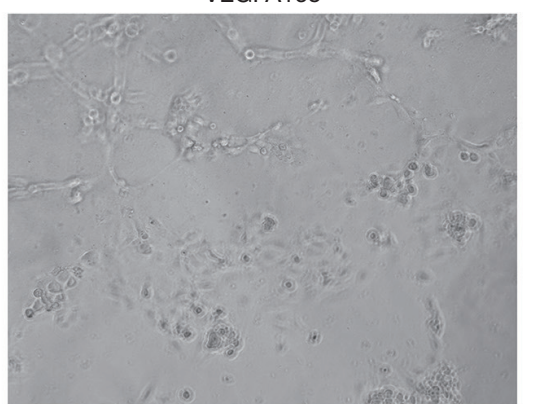

VEGFA165 + NGR2

Figure 4 NGR2 inhibited the cell proliferation and tube formation of pHUVECs induced by VEGFA165. (A,B) The cell viability and proliferation of pHUVECs; (C,D) the tube formation of pHUVECs $(100 \times)$. The data are means \pm SEM of 3 independent experiments performed in triplicate. One-way ANOVA and Student's $t$-test $\left({ }^{*} \mathrm{P}<0.05,{ }^{* *} \mathrm{P}<0.001\right.$ vs. control).

NGR2 + Rap1GAP-si were significantly down-regulated compared with the control group (Figure 6A). In addition, cell viability and cell proliferation in the groups treated with Rap1GAP-si, NGR2 + Rap1GAP-si, and VEGFA165 + NGR2 + Rap1GAP-si were significantly increased compared to the corresponding group untreated with Rap1GAP-si, while the concentrations of intracellular pyruvate and lactic acid decreased (Figure 6B-6E). There were no significant differences in cell viability, cell proliferation, and the concentrations of intracellular pyruvate and lactic acid between the two groups treated with Rap1GAP-si (Figure 6B-6E).
The results demonstrated that NGR2 inhibited the cell proliferation of pHUVECs via targeting Rap1GAP.

\section{NGR2 blocked the Rap1GAP/PI3K/Akt signaling pathway}

The Rap1GAP silencing plasmid and overexpression plasmid were transfected into pHUVECs to explore whether NGR2 regulated angiogenesis via the Rap1GAP and PI3K/ Akt signaling pathway. Compared with the control group, Rap1GAP proteins down-regulated by VEGFA165 were up-regulated in the NGR2 $(10.0 \mathrm{nM})$ group, Rap1GAP-ov group, and Rap1GAP-ov + NGR2 group. Meanwhile, the 
A

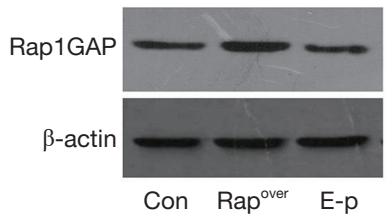

Leg: Rap ${ }^{\text {over: Rap1GAP }}{ }^{\text {over }}$ plasmid; E-p: Empty plasmid.

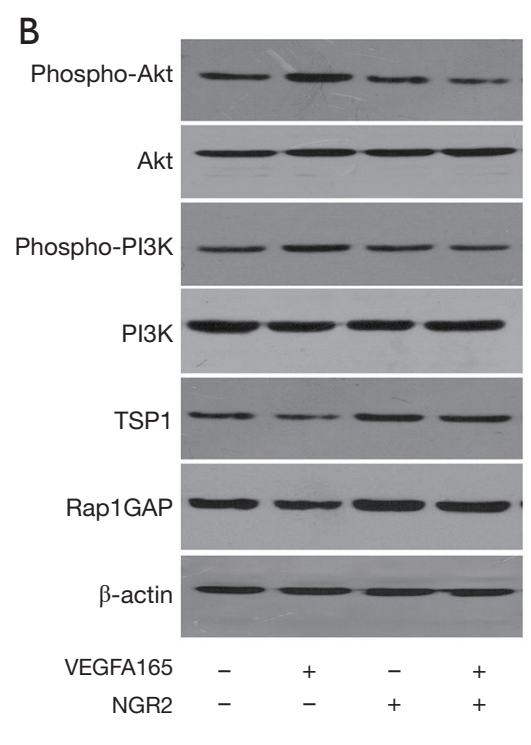

$E$

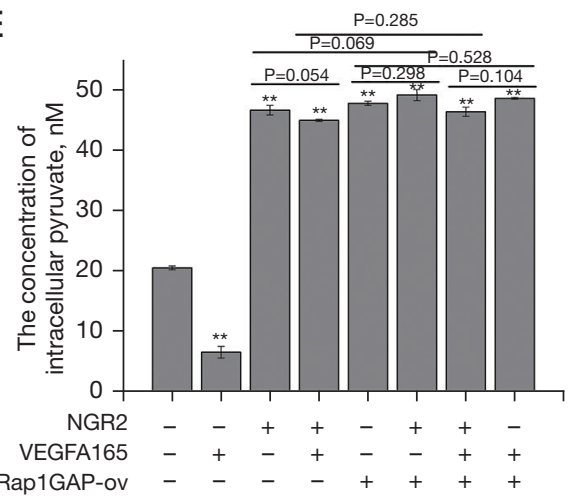

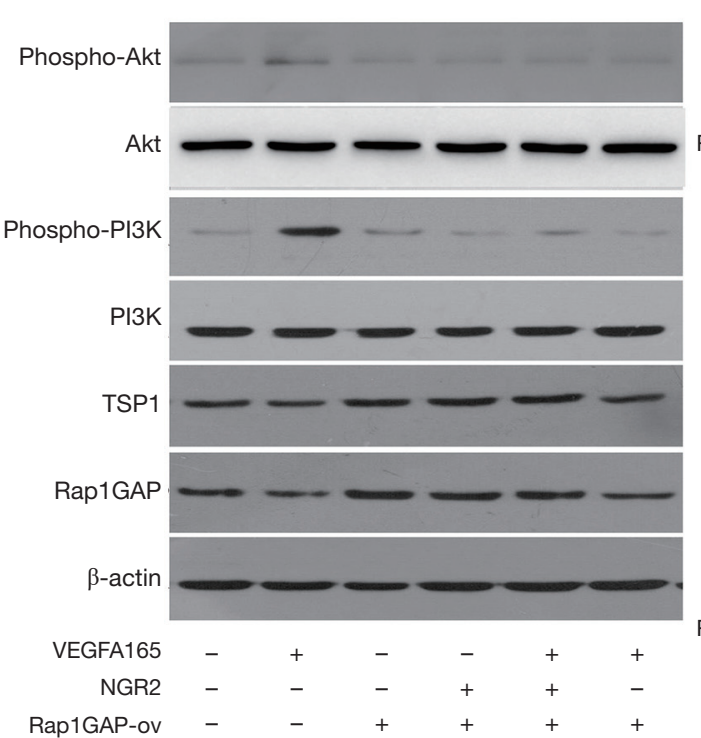

$\mathrm{F}$

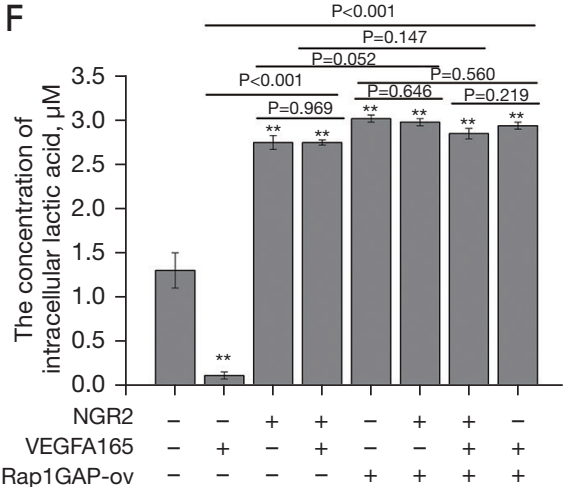

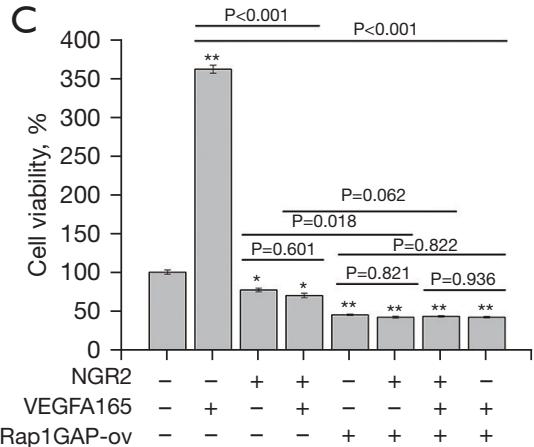
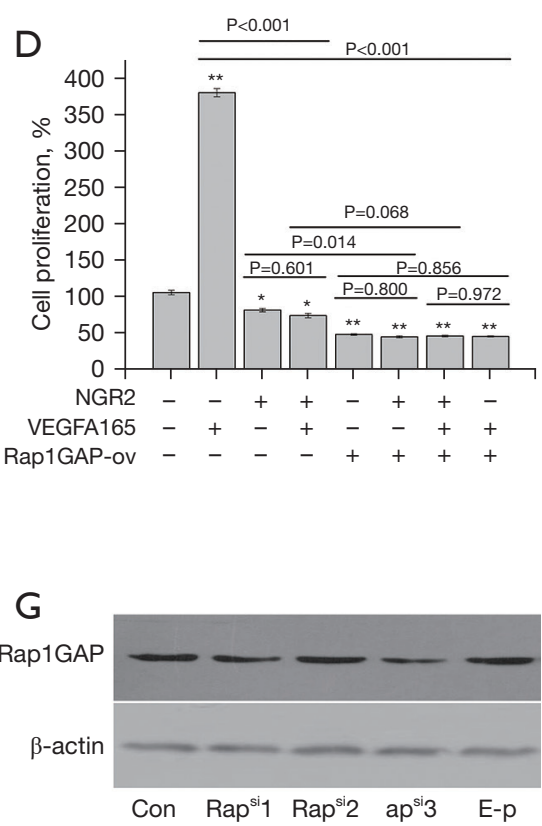

Leg: E-p: Empty plasmid; Rap ${ }^{\text {si }: ~ R a p 1 G A P ~}{ }^{\text {si }}$ plasmid.

Figure 5 Overexpression of Rap1GAP and NGR2 inhibited the cell proliferation of pHUVECs. (A) The gene expression of Rap1GAP in pHUVECs was up-regulated by Rap1GAPover plasmid; (B) the protein expression of Rap1GAP, TSP1, PI3K, phospho-PI3K, Akt, and phospho-Akt in pHUVECs treated with VEGFA165, NGR2, and Rap1GAPover plasmid; (C-F) the cell viability, cell proliferation, and concentration of intracellular pyruvate and lactic acid in pHUVECs treated with VEGFA165, NGR2, and Rap1GAPover plasmid; (G) the expression of Rap1GAP in pHUVECs was down-regulated by Rap1GAPsi plasmid; The data are means \pm SEM of 3 independent experiments performed in triplicate. One-way ANOVA and Student's $t$-test $\left({ }^{*} \mathrm{P}<0.05,{ }^{* *} \mathrm{P}<0.001\right.$ vs. control). 
A
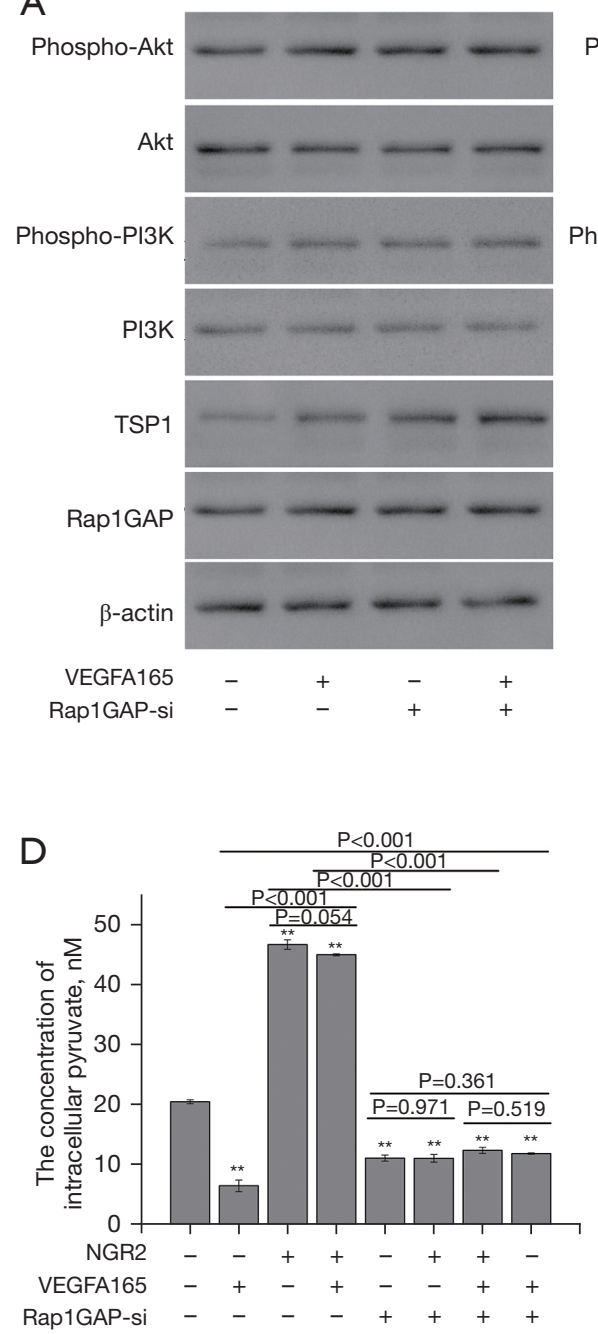

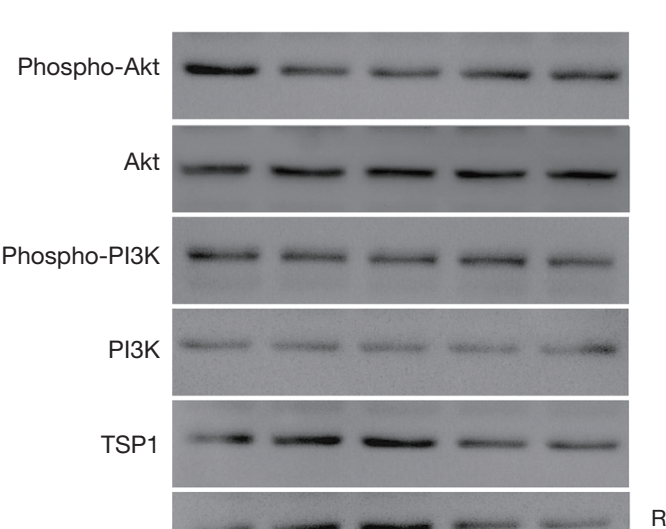

B
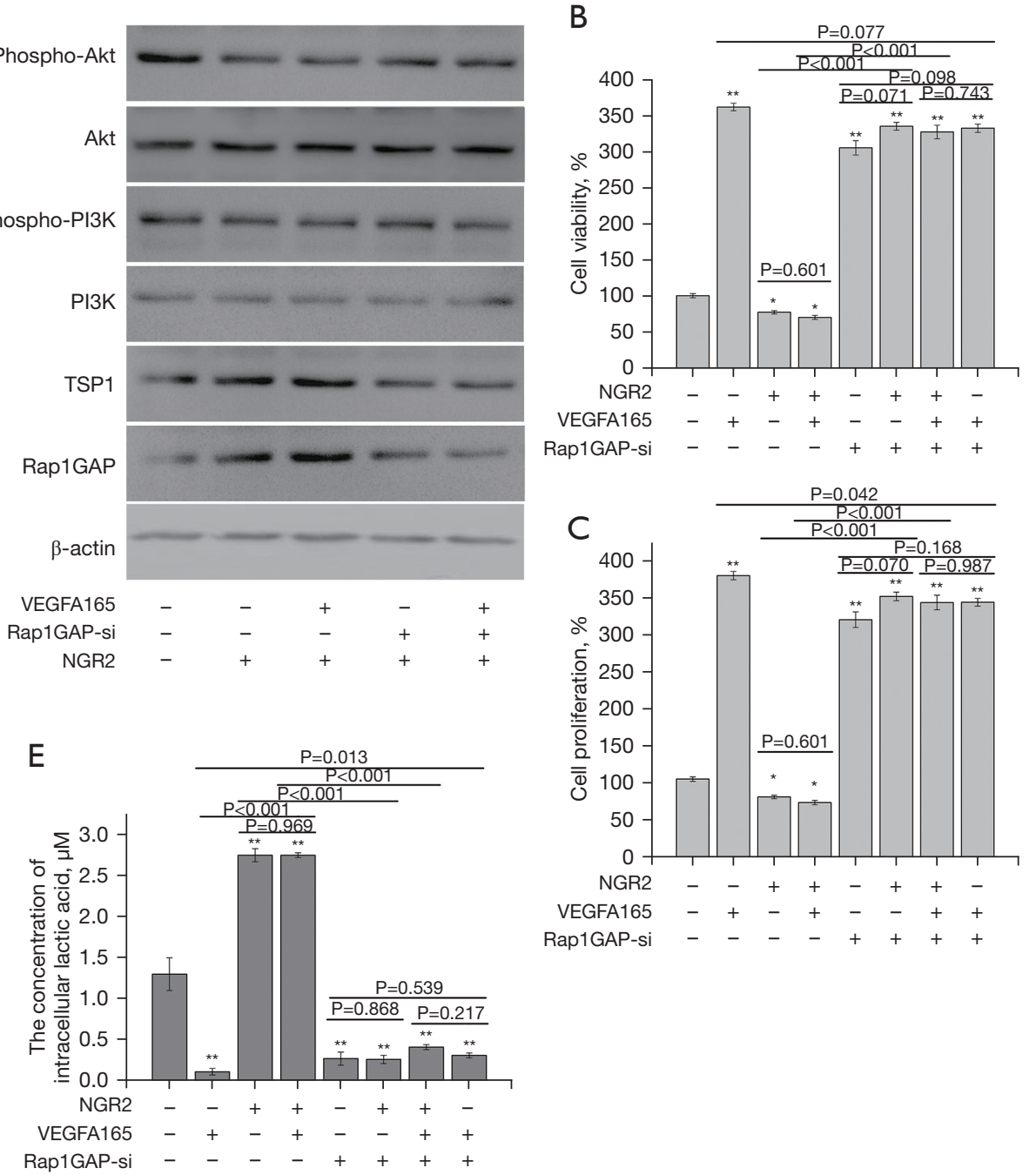

Figure 6 Silencing Rap1GAP promoted the cell proliferation of pHUVECs. (A) The protein expression of Rap1GAP, TSP1, PI3K, phospho-PI3K, Akt, and phospho-Akt in pHUVECs treated with VEGFA165, NGR2, and Rap1GAPsi plasmid; (B-E) the cell viability, cell proliferation, and concentration of intracellular pyruvate and lactic acid in pHUVECs treated with VEGFA165, NGR2, and Rap1GAPsi plasmid. The data are means \pm SEM of 3 independent experiments performed in triplicate. One-way ANOVA and Student's $t$-test $\left({ }^{*} \mathrm{P}<0.05\right.$, ${ }^{* *} \mathrm{P}<0.001$ vs. control).

protein expression levels of phospho-PI3K and phosphoAkt were significantly down-regulated by Rap1GAP1-ov compared with the control group and the empty plasmid (E-p) group, and were up-regulated by $R a p 1 G A P-s i$ (Figure $5 B$ and Figure 6A). However, there were no differences in the protein expression of total PI $3 \mathrm{~K}$ and Akt compared with the control group. In addition, the protein expression of phospho-Akt could be downregulated in the NGR2, NGR2 + LY294002, and LY294002 groups. Meanwhile, there were no differences in the protein expression of phospho-Akt found between the
NGR2 + LY294002 group and LY294002 group. The cell proliferation of pHUVECs was inhibited with the downregulation of phospho-Akt protein expression (Figure $7 A$ ). Furthermore, to explore the regulatory effect of Rap1GAP on the PI3K/Akt signaling pathway, we found that the upregulated phospho-PI3K and phospho-Akt proteins in the Rap1GAP-si group and VEGFA165 group were significantly decreased by LY294002. In addition, there was no difference in the protein expression of phospho-PI3K and phospho-Akt between the LY294002 group and LY294002 + Rap1GAP-ov group (Figure 7B). 
A

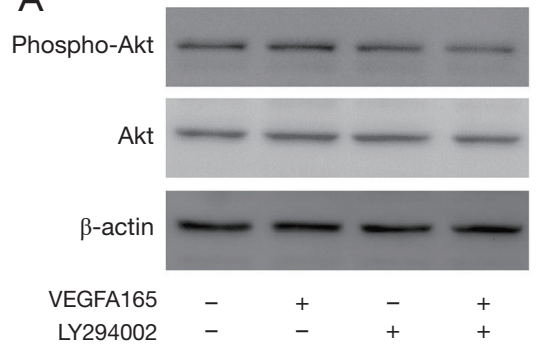

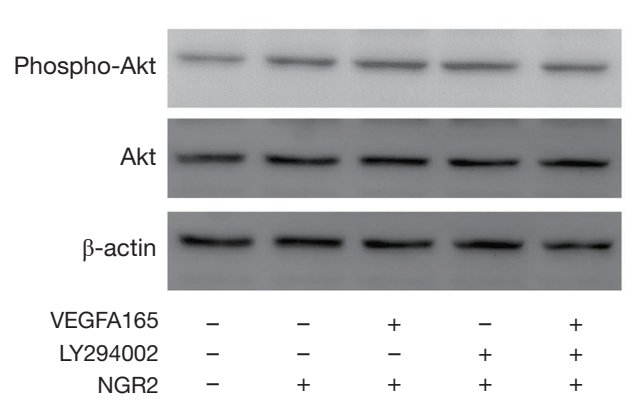

B

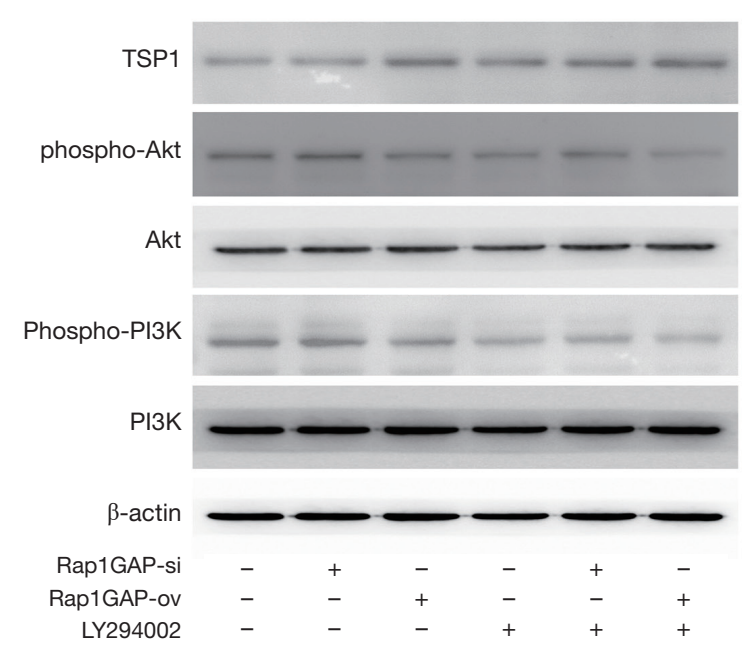

C

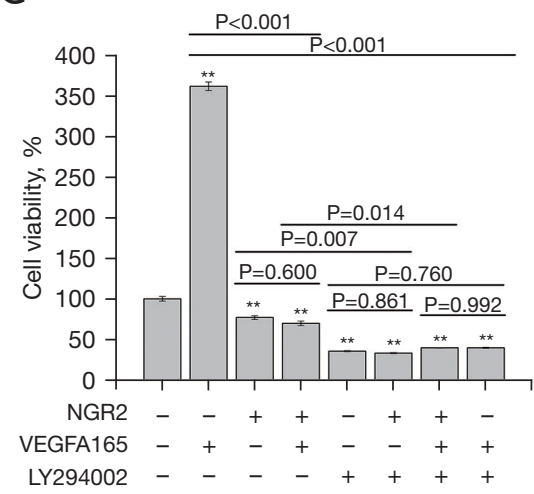

D

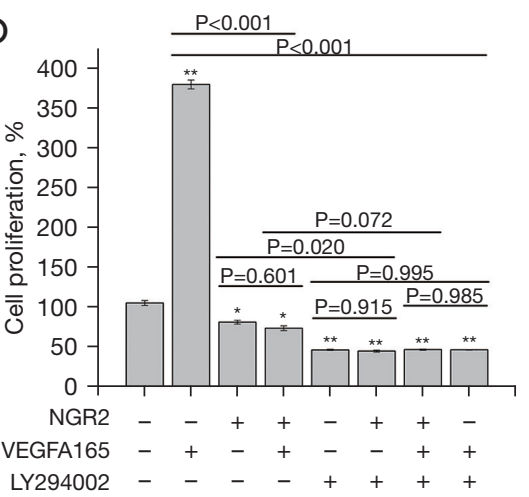

$\mathrm{E}$

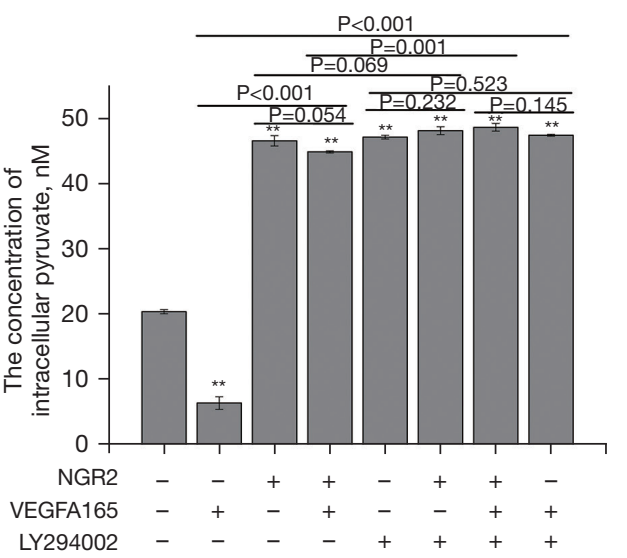

$\mathrm{F}$

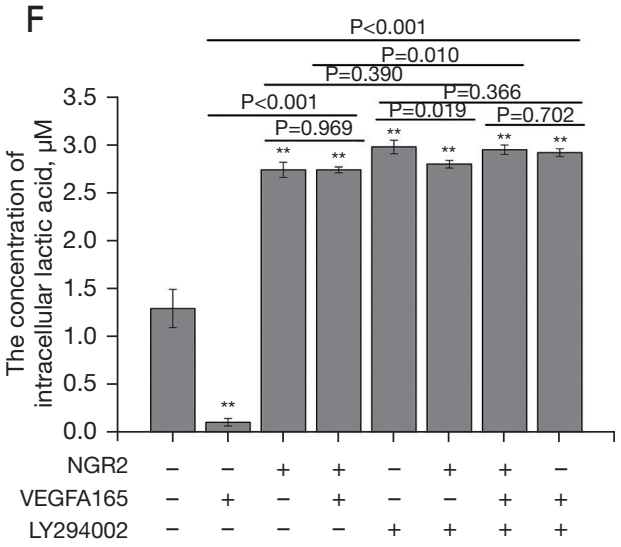

Figure 7 NGR2 blocked the Rap1GAP/PI3K/Akt signaling pathway. (A) The protein expression of phospho-Akt and Akt in the NGR2, NGR2 + LY294002, and LY294002 groups; (B) the expression of proteins in the PI3K/Akt signaling pathway could be regulated by Rap1GAPover plasmid and Rap1GAPsi plasmid, and could also be blocked by LY294002; (C-F) the cell viability, cell proliferation, and concentration of intracellular pyruvate and lactic acid of pHUVECs. The data are means \pm SEM of 3 independent experiments performed in triplicate. One-way ANOVA and Student's $t$-test $\left({ }^{*} \mathrm{P}<0.05,{ }^{* *} \mathrm{P}<0.001\right.$ vs. control). 


\section{Page 12 of 14}

The cell viability and cell proliferation of pHUVECs in the Rap1GAP-si group and VEGFA165 group were significantly inhibited by LY294002, while the concentration of intracellular pyruvate and lactic acid increased (Figure $7 C-7 F)$.

\section{Discussion}

PN has been shown to promote angiogenesis, regulate metabolism, and reduce the inflammatory response (17). Our previous research also found that PN could significantly promote angiogenesis and reduce vascular inflammatory injuries in colitis $(3,4)$. Indeed, some saponins in PN, which are important active chemical components including ginsenosides and notoginsenosides, have appeared to lack toxicity in both animals and humans (5). Therefore, $\mathrm{PN}$ is regarded as a healthpreserving drug that can protect the structure and function of blood vessels in daily life. $\mathrm{PN}$ is a traditional medicine that has the effect of promoting angiogenesis and antiangiogenesis. However, the anti-angiogenesis effect and active ingredients of $\mathrm{PN}$ have been seriously neglected in studies.

Notoginsenoside is the main active component of PN which regulates angiogenesis and inflammation in vivo and vitro $(18,19)$. Among them, NGR2 is an important active component in PN (20). Hu et al. found that NGR2 could prevent neuronal apoptosis and inflammation via miR-27a/ SOX8/-catenin axis in Alzheimer's disease (8). However, the effect of NGR2 on angiogenesis is still unclear. Our results showed that NGR2 could inhibit the cell viability, cell proliferation, and tubulogenesis of pHUVECs and induce colonic mucosal and microvascular injuries in the colons of SD rats. Meanwhile, the protein expression of HIF- $1 \alpha$ and the content of intracellular pyruvate and lactic acid were significantly increased in the colonic mucosa of rats treated with NGR2. Therefore, NGR2 could be a negative regulator of angiogenesis and enhance hypoxia in the colonic mucosa.

Angiogenesis is a complex physiological and pathological process. It is well known that VEGFA, an important subtype of VEGF, plays an important role in regulating the cell proliferation and tube formation of VECs (21). In addition, VEGFA consists of many isoforms, such as VEGFA121 and VEGFA165, among others (13). The results of previous studies showed that the abnormal ratio of VEGFA165/VEGFA121 is closely correlated with vascular injuries in a variety of diseases, such as
Tao et al. NGR2 induces colonic microvascular injuries in colitis

ulcerative colitis and cancers $(12,13)$. VEGFR2 is the major receptor implicated in process of regulating cell viability, cell proliferation, cell migration, and VP stimulated by VEGFA (22). Our previous study found that the abnormal ratio of VEGFA165/VEGFA121 was closely correlated with colonic mucosal microvascular injuries in UC rats, and the activation of different phosphorylation sites of VEGFR2 led to VEGFA12 1 and VEGFA165 exerting opposite effects on angiogenesis (12). Besides, VEGFA also plays an important role in regulating glucose metabolism in VECs. VEGFA could reduce intracellular glycolysis and the protein expression of HIF-1 $\alpha$ in HUVECs (23). In the present study, after being treated with NGR2 for 7 days, the contents of VEGFA165 and VEGFA121 increased significantly, accompanied by the abnormal ratio of VEGFA165/VEGFA121 in rats treated with NGR2, and colonic mucosal and microvascular injuries also increased as hypoxia increased. It was also found that NGR2 could significantly inhibit the effects of VEGFA on regulating the cell proliferation and tubulogenesis of HUVECs and increase the intracellular glycolysis, which could cause microvascular injuries and hypoxia in tissue.

The PI3K/Akt signaling pathway is an important signaling pathway which has been shown to regulate cell proliferation, metastasis, and differentiation, among other processes (24). In addition, related studies found that VEGFA could promote angiogenesis via activating the PI3K/Akt signaling pathway (25). In this study, LY294002 was used as a PI3K/Akt signaling pathway inhibitor to investigate the mechanism of NGR2 in inhibiting angiogenesis. We found that NGR2 was an analog of the PI3K inhibitor which inhibited protein expression of phospho-Akt, and was similar to LY294002. Meanwhile, the cell viability and cell proliferation of pHUVECs were inhibited by LY294002 and NGR2. In addition, the signal transduction of the PI3K/Akt signaling pathway could be regulated by multiple signaling molecules, such as VEGFR2, PTEN, and Rap1GAP, among others $(26,27)$. Of these, overexpression of Rap1GAP1, a GTPase-activating protein inhibiting Rap1 expression, effectively regulated angiogenesis via inhibiting cell proliferation, cell migration, tubulogenesis, and integrin-dependent adhesion $(12,28)$. Besides, TSP1, a major protein driving endothelial cell senescence, could also inhibit angiogenesis directly by interacting with VEGF or indirectly by combining with TSP1 receptors on endothelial cells (29). The present study found that NGR2 could significantly up-regulate the protein expression of Rap1GAP and TSP1 in the colonic 
mucosa of rats and pHUVECs, and enhanced intracellular glycolysis and hypoxia. Furthermore, the effect of NGR2 on anti-angiogenesis could be recovered via downregulating the expression of Rap1GAP in pHUVECs, which could inhibit the PI3K/Akt signaling pathway as well as LY294002. Overall, NGR2 inhibited angiogenesis and enhanced intracellular glycolysis via activating the Rap1GAP/PI3K/Akt signaling pathway.

In summary, NGR2 was an analog of the Rap1GAP inhibitor. It could inhibit the cell proliferation, tube formation, and glucose aerobic oxidation of HUVECs via regulating the Rap1GAP/PI3K/Akt signaling pathway. However, there are still some deficiencies in this study. For example, there is a lack of in vivo verification of the regulatory effects of NGR2 on angiogenesis using RapGAP gene intervention animal models. Additionally, although NGR2 could promote the intracellular glycolysis of HUVECs, the mechanism remains unclear.

\section{Acknowledgments}

The authors thank Wanlin Yu and Huan Zheng who helped with the study and Dr. Chen Dong who provided help in biostatistics.

Funding: The study was supported by the National Natural Science Foundation of China (No. 81904106) and Major Scientific Research Projects at Provincial Level in Guangdong General University (No. 2018KQNCX044).

\section{Footnote}

Reporting Checklist: The authors have completed the ARRIVE reporting checklist. Available at https://dx.doi. org/10.21037/atm-21-5898

Data Sharing Statement: Available at https://dx.doi. org/10.21037/atm-21-5898

Conflicts of Interest: All authors have completed the ICMJE uniform disclosure form (available at https://dx.doi. org/10.21037/atm-21-5898). The authors have no conflicts of interest to declare.

Ethical Statement: The authors are accountable for all aspects of the work in ensuring that questions related to the accuracy or integrity of any part of the work are appropriately investigated and resolved. Animal experiments were performed under a project license (No.
SZY201612006) granted by the Institutional Animal Care and Use Committee of Shanghai University of TCM, in compliance with the Institutional Animal Care and Use Committee of Shanghai University of TCM's guidelines for the care and use of animals.

Open Access Statement: This is an Open Access article distributed in accordance with the Creative Commons Attribution-NonCommercial-NoDerivs 4.0 International License (CC BY-NC-ND 4.0), which permits the noncommercial replication and distribution of the article with the strict proviso that no changes or edits are made and the original work is properly cited (including links to both the formal publication through the relevant DOI and the license). See: https://creativecommons.org/licenses/by-nc-nd/4.0/.

\section{References}

1. Michaels J 5th, Dobryansky M, Galiano RD, et al. Topical vascular endothelial growth factor reverses delayed wound healing secondary to angiogenesis inhibitor administration. Wound Repair Regen 2005;13:506-12.

2. Men SY, Huo QL, Shi L, et al. Panax notoginseng saponins promotes cutaneous wound healing and suppresses scar formation in mice. J Cosmet Dermatol 2020;19:529-34.

3. Wang S, Tao P, Zhao L, et al. Panax notoginseng Promotes Repair of Colonic Microvascular Injury in Sprague-Dawley Rats with Experimental Colitis. Evid Based Complement Alternat Med 2018;2018:4386571.

4. Wang SY, Tao P, Hu HY, et al. Effects of initiating time and dosage of Panax notoginseng on mucosal microvascular injury in experimental colitis. World J Gastroenterol 2017;23:8308-20.

5. Xu C, Wang W, Wang B, et al. Analytical methods and biological activities of Panax notoginseng saponins: Recent trends. J Ethnopharmacol 2019;236:443-65.

6. Zhang L, Li Y, Ma X, et al. Ginsenoside Rg1Notoginsenoside R1-Protocatechuic Aldehyde Reduces Atherosclerosis and Attenuates Low-Shear Stress-Induced Vascular Endothelial Cell Dysfunction. Front Pharmacol 2021;11:588259.

7. Liu J, Jiang C, Ma X, et al. Notoginsenoside Fc attenuates high glucose-induced vascular endothelial cell injury via upregulation of PPAR- $\gamma$ in diabetic Sprague-Dawley rats. Vascul Pharmacol 2018;109:27-35.

8. Hu Y, Wu L, Jiang L, et al. Notoginsenoside R2 reduces A 25 -35-induced neuronal apoptosis and inflammation via 


\section{Page 14 of 14}

miR-27a/SOX8/ $\beta$-catenin axis. Hum Exp Toxicol 2021.

[Epub ahead of print].

9. Li X, Zhang $\mathrm{Y}$, Hong $Z$, et al. Transcriptome Profiling Analysis Reveals the Potential Mechanisms of Three Bioactive Ingredients of Fufang E'jiao Jiang During Chemotherapy-Induced Myelosuppression in Mice. Front Pharmacol 2018;9:616.

10. Gao Y, Zhao D, Xie WZ, et al. Rap1GAP Mediates Angiotensin II-Induced Cardiomyocyte Hypertrophy by Inhibiting Autophagy and Increasing Oxidative Stress. Oxid Med Cell Longev 2021;2021:7848027.

11. Li W, Jin B, Cornelius LA, et al. Inhibitory effects of Rap1GAP overexpression on proliferation and migration of endothelial cells via ERK and Akt pathways. J Huazhong Univ Sci Technolog Med Sci 2011;31:721-7.

12. Shiying $W$, Boyun $S$, Jianye $Y$, et al. The Different Effects of VEGFA121 and VEGFA165 on Regulating Angiogenesis Depend on Phosphorylation Sites of VEGFR2. Inflamm Bowel Dis 2017;23:603-16.

13. Dallinga MG, Habani YI, Schimmel AWM, et al. The Role of Heparan Sulfate and Neuropilin 2 in VEGFA Signaling in Human Endothelial Tip Cells and Non-Tip Cells during Angiogenesis In Vitro. Cells 2021;10:926.

14. Prete A, Lo AS, Sadow PM, et al. Pericytes Elicit Resistance to Vemurafenib and Sorafenib Therapy in Thyroid Carcinoma via the TSP-1/TGF $\beta 1$ Axis. Clin Cancer Res 2018;24:6078-97.

15. Sun B, Yuan J, Wang S, et al. Qingchang Suppository Ameliorates Colonic Vascular Permeability in DextranSulfate-Sodium-Induced Colitis. Front Pharmacol 2018;9:1235.

16. Maughan RJ. A simple, rapid method for the determination of glucose, lactate, pyruvate, alanine, 3-hydroxybutyrate and acetoacetate on a single 20-mul blood sample. Clin Chim Acta 1982;122:231-40.

17. Duan L, Xiong X, Hu J, et al. Panax notoginseng Saponins for Treating Coronary Artery Disease: A Functional and Mechanistic Overview. Front Pharmacol 2017;8:702.

18. Zhu T, Xie WJ, Wang L, et al. Notoginsenoside R1 activates the NAMPT-NAD+-SIRT1 cascade to promote postischemic angiogenesis by modulating Notch signaling. Biomed Pharmacother 2021;140:111693.

19. Lei W, Li X, Li L, et al. Compound Danshen Dripping Pill ameliorates post ischemic myocardial inflammation through synergistically regulating MAPK, PI3K/ AKT and PPAR signaling pathways. J Ethnopharmacol 2021;281:114438.

20. Li X, Wang Y, Fan Z, et al. High-level sustainable
Tao et al. NGR2 induces colonic microvascular injuries in colitis

production of the characteristic protopanaxatriol-type saponins from Panax species in engineered Saccharomyces cerevisiae. Metab Eng 2021;66:87-97.

21. Trapp V, Parmakhtiar B, Papazian V, et al. Anti-angiogenic effects of resveratrol mediated by decreased VEGF and increased TSP1 expression in melanoma-endothelial cell co-culture. Angiogenesis 2010;13:305-15.

22. Peach CJ, Mignone VW, Arruda MA, et al. Molecular Pharmacology of VEGF-A Isoforms: Binding and Signalling at VEGFR2. Int J Mol Sci 2018;19:1264.

23. Martí-Díaz R, Montenegro MF, Cabezas-Herrera J, et al. Acriflavine, a Potent Inhibitor of HIF-1 $\alpha$, Disturbs Glucose Metabolism and Suppresses ATF4-Protective Pathways in Melanoma under Non-Hypoxic Conditions. Cancers (Basel) 2020;13:102.

24. Wu S, Chen M, Huang J, et al. ORAI2 Promotes Gastric Cancer Tumorigenicity and Metastasis through PI3K/ Akt Signaling and MAPK-Dependent Focal Adhesion Disassembly. Cancer Res 2021;81:986-1000.

25. Zhang G, Hu Y, Yuan W, et al. miR-519d-3p Overexpression Inhibits P38 and PI3K/AKT Pathway via Targeting VEGFA to Attenuate the Malignant Biological Behavior of Non-Small Cell Lung Cancer. Onco Targets Ther 2020;13:10257-66.

26. Liu C, He L, Wang J, et al. Anti-angiogenic effect of Shikonin in rheumatoid arthritis by downregulating PI3K/ AKT and MAPKs signaling pathways. J Ethnopharmacol 2020;260:113039.

27. Pei H, Wang W, Zhao D, et al. G Protein-Coupled Estrogen Receptor 1 Inhibits Angiotensin II-Induced Cardiomyocyte Hypertrophy via the Regulation of PI3KAkt-mTOR Signalling and Autophagy. Int J Biol Sci 2019;15:81-92.

28. Carmona G, Göttig S, Orlandi A, et al. Role of the small GTPase Rap1 for integrin activity regulation in endothelial cells and angiogenesis. Blood 2009;113:488-97.

29. Bazzazi H, Zhang Y, Jafarnejad M, et al. Computer Simulation of TSP1 Inhibition of VEGF-Akt-eNOS: An Angiogenesis Triple Threat. Front Physiol 2018;9:644.

(English Language Editor: C. Betlazar-Maseh)

Cite this article as: Tao P, Lin J, Zhang B, Wang S. Notoginsenoside R2 induces colonic microvascular injuries via regulating the Rap1GAP/PI3K/Akt signaling pathway. Ann Transl Med 2021;9(23):1743. doi: 10.21037/atm-21-5898 